\title{
Thermal and Structural Characterization of Two Commercially Available Technical Lignins for High-value Applications
}

Tanvir A. Amit ${ }^{\mathbf{a}}$, Ranen Roy ${ }^{\mathbf{b}}$, and Douglas E. Raynie*

Department of Chemistry and Biochemistry, South Dakota State University, Brookings, SD 57007, USA

Tanvir.Amit@jacks.sdtsate.edu ${ }^{\mathrm{a}}$; Ranen.Roy@sdstate.edu ${ }^{\mathrm{b}}$; Douglas.Raynie@sdstate.edu*

\section{*Corresponding Author:}

Tel: +1 6056885151

E-mail: Douglas.Raynie@sdstate.edu

\begin{abstract}
Lignin is a complex polyaromatic macromolecule and a potential source of various sustainable materials and feedstock chemicals. To this end, researchers have made some considerable efforts in the high-value applications of lignin, even though there is a limited success so far. This is mainly because the exact structure of native lignin is still virtually unknown due to its highly heterogeneous nature. Besides, technical lignin undergoes unintended structural modifications during the chemical pulping and extraction processes making its final structure even more complicated. Therefore, understanding the lignin structure and its macromolecular characteristics is essential for its proper utilization. In this study, two technical lignins, such as indulin AT and alkali-treated lignin, were investigated for thermal and structural characterization. Various thermal behaviors were studied using thermogravimetric analysis (TGA) and differential scanning calorimetry (DSC). Indulin AT was found to be thermally more stable compared to alkali lignin. Structural characterization was performed using attenuated total reflectance-Fourier transform infrared (ATR-FTIR) spectroscopy and proton nuclear magnetic resonance spectroscopy $\left({ }^{1} \mathrm{H}\right.$ NMR). Cupric oxide oxidation was utilized to selectively degrade the lignin into its monomers (H/G/S-moieties), which were identified with GC-MS. The results suggested that the selected lignins are mainly composed of G-type monomers. The detailed characterization studies also revealed some minor structural differences between the two lignins due to their respective delignification process. Indulin AT underwent higher structural modifications due to the harsher delignification process and hinted to show more recalcitrance toward depolymerization than alkali lignin.
\end{abstract}

Keywords: Technical lignin; Lignin characterization; Lignin application; GC-MS; Indulin AT; Alkali lignin 


\section{Introduction}

Many commodity products such as energy, materials, chemicals, and transportation fuels that we use today are mainly produced from petroleum resources [1, 2]. However, unfortunately, the world's fossil carbon sources are not going to last forever. The depletion of petroleum reserves has encouraged the global community to lean towards sustainable development by replacing fossil carbon sources. To this end, lignocellulosic biomass offers many possibilities to produce both energies and various value-added chemicals due to its chemical composition, abundant availability, and relatively low cost [3]. Lignocellulosic biomass mainly consists of three major polymeric components: cellulose, hemicellulose, and lignin; whereas lignin is the second most abundant natural polymer on earth after cellulose making up 15-35\% of the total weight of the biomass [4,5].

Most lignins in the world are produced as byproducts, mainly by the pulping and bioethanol industries, where lignin is removed from the wood or biomass feedstock to produce the target products [6]. Lignin isolated from the industrial processes is called technical lignin. Technical lignins can be categorized into two major types based on the extraction processes: (i) sulfur lignin and (ii) sulfur-free (or low sulfur content) lignin [7]. Kraft lignins and lignosulfonates are the products of commercial pulping processes and fall into the first category, whereas sulfur-free or low sulfur content lignins include soda, alkaline or organosolv lignins, which are the products of bioethanol production processes [8]. The worldwide estimated production of technical lignin is about 100 million tons per year [9].

Nevertheless, lignin is often regarded as a low-value product as the bulk of the industrial lignins are either burned onsite to provide process heat or goes to waste; only about 1-2\% of the lignin is used for making other products [10]. However, owing to the highly aromatic lignin structure, researchers have made many attempts in recent years to utilize and convert the surplus industrial lignins into more profitable monomeric aromatics. To that end, lignins are either depolymerized or utilized as chemical feedstocks to produce emerging and valuable biomaterials such as carbon nanofiber, organic membrane, polyurethane, and phenol-formaldehyde resin [1114]. Such high-value lignin applications have motivated scientists worldwide to understand this complex molecule further and continue its novel utilization to stimulate the worldwide bio-based economy in the near future. 
Lignin is a three-dimensional heterogeneous biopolymer bound primarily with the polysaccharides such as cellulose and hemicellulose and located in the secondary cell walls of the vascular plants. The chemical structure of lignin is very complex, and its backbone is mainly composed of three methoxylated phenylpropane units (see Fig. 1). These monomeric units or major precursors are aromatic alcohols, namely p-coumaryl alcohol (p-hydroxyphenyl $(\mathrm{H})$ moiety), coniferyl alcohol (guaiacyl (G) moiety), and sinapyl alcohol (syringyl (S) moiety) [15]. The phenylpropane units randomly polymerize with each other via dehydrogenative enzymeinitiated free-radical polymerization reaction and form a three-dimensional cross-linked amorphous structure [16].

The composition and combination of these monomeric units (H/G/S-moiety) in the native lignin structure vary depending on the biomass sources, plant tissue types, age of the plants, $\mathrm{pH}$ of the soil, and weather that creates a wide structural diversity of lignin biopolymers [17, 18]. Besides, the native structure cannot be retained as it undergoes unintended structural modifications during the chemical pulping processes due to disruption of the ester and ether linkages [19]. So, not just the botanical sources, delignification (pulping) and extraction processes also influence the lignin structure, purity, and properties [20, 21]. Therefore, detail and careful characterization of technical lignins obtained from different pulping processes such as kraft, soda, lignosulfonate, organosolv, or lignoboost is essential because different processes utilize different process chemistry and physical conditions (temperature, pressure, time) that impart processspecific heterogeneity in the final structure of the technical lignin.

This study aimed to perform thermal and structural characterization of two commercially available lignins, namely indulin AT and alkali lignin. Indulin AT is a high sulfur content (7.0$3.0 \%)$ kraft lignin obtained from the black liquor of linerboard pulp, while alkali-treated lignin is a low sulfur content $(<4.0 \%)$ lignin. A thorough characterization of these technical lignins will help researchers better understand the process-specific structural heterogeneity of the selected lignins and unlock their potential applicability as feedstock to make various novel bio-based products.

In this study, thermal characterization of the selected lignin samples was performed using thermogravimetric analysis (TGA) and differential scanning calorimetry (DSC). TGA study helped elucidate the course of thermal degradations and compare the thermal stability of the selected lignins. DSC analysis was able to reveal thermal properties such as glass transition $\left(T_{\mathrm{g}}\right)$ 
and melting temperatures. Overall thermal properties obtained from the two techniques also provided insights about possible structural modifications of the lignin samples during the pulping process. Chemical degradative study such as cupric oxide $(\mathrm{CuO})$ oxidation followed by gas chromatography-mass spectrometry (GC-MS) was performed to identify the monomeric degradation products, which are indicative of the presence of specific monolignol(s) (H/G/S-unit) that the lignin samples are mainly composed of. Attenuated total reflectance (ATR) in conjunction with traditional Fourier transform infrared (FTIR) spectroscopy revealed various typical functional groups and characteristic absorption bands specific to the H/G/S-moiety in the lignin samples. Finally, proton nuclear magnetic resonance $\left({ }^{1} \mathrm{H}\right.$ NMR) spectroscopy was utilized to investigate the differences between the lignin samples' overall structure employing quantitative measurement of some key chemical functionalities and inter-unit linkages.

\section{Materials and Methods}

\subsection{Materials}

The characterization study exploited two technical lignins, namely indulin AT and alkali lignin, that were commercially available to purchase from MeadWestvaco Corporation (Charleston, SC, USA) and Sigma-Aldrich Inc. (St. Louis, MO, USA), respectively. The chemicals required for the cupric oxide oxidation experiment such as copper (II) oxide (99.999\% trace metals basis) and hydrochloric acid (reagent grade, 37\%) were purchased from SigmaAldrich, and sodium hydroxide (certified ACS grade) and dichloromethane (certified ACS grade) were purchased from Fisher Scientific (Hampton, NH, USA). The lignin degradation compounds such as guaiacol (99.1\%), vinyl guaiacol ( $\geq 98.0 \%$ ), vanillin (99.7\% $\pm 0.3 \%$ ), acetovanillone ( $\geq$ $98.0 \%$ ), homovanillic acid ( $\geq 98.0 \%$ ), and $p$-cresol ( $\geq 99.0 \%)$ were purchased from Sigma-

Aldrich and used as analytical standards in the GC-MS analysis. Deuterated solvent (DMSO-d6) and internal standard, 1,3,5-trioxane for the NMR study were purchased from Sigma-Aldrich.

\subsection{Thermal characterization}

\subsubsection{Thermogravimetric analysis (TGA)}

TGA of indulin AT and alkali lignin was carried out using a Perkin Elmer Pyris-1 instrument (Waltham, MA, USA). About $10 \mathrm{mg}$ of the respective lignin sample was weighed in a platinum pan for the analysis. The oven was programmed from room temperature to $900^{\circ} \mathrm{C}$ at a constant 
heating rate of $10{ }^{\circ} \mathrm{C} / \mathrm{min}$. Nitrogen $\left(\mathrm{N}_{2}\right)$ at a $20 \mathrm{~mL} / \mathrm{min}$ flow was used to prevent any probable combustion of the lignin samples with air.

\subsubsection{Differential scanning calorimetry (DSC)}

DSC measurements of the selected lignin samples were performed on a DSC Q-200 differential scanning calorimeter obtained from TA Instruments (New Castle, DE, USA). About $10 \mathrm{mg}$ of the lignin sample was weighed in a $\mathrm{T}_{\text {zero }}$ aluminum hermetic pan and analyzed under a nitrogen $\left(\mathrm{N}_{2}\right)$ atmosphere. $\mathrm{N}_{2}$ flow was $20 \mathrm{~mL} / \mathrm{min}$ throughout the experiment. The lignin samples were annealed at $60{ }^{\circ} \mathrm{C}$ for $24 \mathrm{~h}$ in a vacuum oven before the analysis to remove any presence of water and obtain a perceptible glass transition $\left(\mathrm{T}_{\mathrm{g}}\right)$ on the DSC heating curve. During the analysis, the lignin samples were heated from 20 to $110^{\circ} \mathrm{C}$ at a heating rate of $10{ }^{\circ} \mathrm{C} / \mathrm{min}$ and cooled to $20^{\circ} \mathrm{C}$ at the same rate to remove moisture and negate any previous thermal history. The lignin samples were heated again from 20 to $400{ }^{\circ} \mathrm{C}$ at a constant heating rate of $15{ }^{\circ} \mathrm{C} / \mathrm{min}$, and the scan was recorded and analyzed with the TA-Universal Analysis 2000 software to determine $\mathrm{T}_{\mathrm{g}}$, melting, and decomposition temperature.

\subsection{Structural characterization}

\subsubsection{Cupric oxide (CuO) oxidation study}

The Pearl method with minor modifications was used for the $\mathrm{CuO}$ oxidation of the lignin samples [22]. $100 \mathrm{mg}$ of the respective lignin sample and $1.0 \mathrm{~g}$ of $\mathrm{CuO}$ were taken in a $100-\mathrm{mL}$ three-neck round-bottom flask. The round bottom flask was degassed and filled with nitrogen gas to maintain an inert atmosphere inside the flask. Then $15 \mathrm{~mL}$ of $2.0 \mathrm{M}$ sodium hydroxide $(\mathrm{NaOH})$ was injected into the flask, and the mixture was stirred at room temperature until the lignin mixed well with $\mathrm{NaOH}$. The reaction mixture was then refluxed at $175^{\circ} \mathrm{C}$ for $2.5 \mathrm{~h}$ using water cooling and uniform stirring. After the reaction, the oxidation mixture was cooled to room temperature and filtered to remove insoluble particles. The resultant filtrate was then acidified to $\mathrm{pH} 1-2 \mathrm{using}$ concentrated $\mathrm{HCl}$. More (unreacted) lignin precipitated during this time, and hence, the oxidation mixture was filtered once again. Liquid-liquid extraction was performed three times with 15-mL dichloromethane (DCM), and the extracted DCM layers were combined and evaporated by flowing nitrogen gas over them. The crude oxidation products were dissolved in six mL of DCM and used as a stock solution for further analysis in the gas chromatography-mass spectrometry (GC-MS). 
2.3.1.1. Gas chromatography-mass spectrometry (GC-MS) analysis of the CuO degradation products

Agilent 7890A (New Castle, DE, USA) gas chromatograph equipped with an Agilent 5975C triple-axis mass detector with electron-impact ionization was used for the GC-MS analysis. An injection volume of $2 \mu \mathrm{L}$ was used with splitless mode with an injection temperature of $250^{\circ} \mathrm{C}$. A septum purge flow of $3 \mathrm{~mL} / \mathrm{min}$ was used with a glass-wool packed straight liner. An Agilent DB-5 (250 $\mu \mathrm{m}$ x 30 m) 5\%-phenyl-methylpolysiloxane capillary column was used. The initial temperature of the GC column was set at $60^{\circ} \mathrm{C}$, held for $1 \mathrm{~min}$, then ramped to $250^{\circ} \mathrm{C}$ at $10^{\circ} \mathrm{C} /$ min. Hydrogen at a flow of $1.2 \mathrm{~mL} / \mathrm{min}$ was used as the carrier gas. The MS analysis was performed in full-scan mode with a range of $\mathrm{m} / \mathrm{z} 50-600$, and the Agilent ChemStation software was used to autotune the mass detector. The NIST database and authentic standards were used to identify the structural moieties of the lignin samples.

\subsubsection{Attenuated total reflectance-Fourier transform infrared spectroscopy (ATR-FTIR)}

A Nicolet 380 spectrometer from Thermo-Fisher Scientific (Waltham, MA, USA) was used in the attenuated total reflectance mode to obtain the FTIR spectra of the selected lignin samples. The measurement resolution was set at $4 \mathrm{~cm}^{-1}$, and the spectra were collected in the range of 4000 to $400 \mathrm{~cm}^{-1}$ with 128 co-added scans. The spectra of the selected lignins were processed using EZ OMNIC software.

\subsubsection{The proton nuclear magnetic resonance $\left({ }^{1} H\right.$ NMR) spectroscopy}

The ${ }^{1} \mathrm{H}$ NMR spectra of the indulin AT and alkali lignin were recorded on a Bruker $400 \mathrm{MHz}$ spectrometer (Billerica, MA, USA) equipped with a QNP 5-mm probe at $400 \mathrm{MHz}$ and $295 \mathrm{~K}$. DMSO- $\mathrm{d}_{6}$ was used as a solvent, and 1,3,5-trioxane was used as an internal standard (IS). About $25 \mathrm{mg}$ of the lignin sample was taken with $0.044 \mathrm{mmol}$ IS/0.5-mL DMSO- $\mathrm{d}_{6}$. The parameters for the ${ }^{1} \mathrm{H}$ NMR experiment were $90^{\circ}$ pulse angle, 2-s pulse delay, and 512 scans. All the ${ }^{1} \mathrm{H}$ NMR spectra were analyzed using Topspin 1.3 software.

\section{Results and discussions}

\subsection{Thermal analysis}

\subsubsection{Thermogravimetric analysis (TGA)}


TGA study of indulin AT and alkali lignin revealed their thermal behavior, such as pyrolytic degradation at various temperature ranges, corresponding \%weight loss, and thermal stability. TGA also provided insights into the possible structural modifications of the selected lignins due to the pulping processes. TGA thermograms were obtained in the form of thermogravimetric (TG) and derivative thermogravimetric (DTG) curves that are illustrated in Fig. 2(A) and (B), respectively. Table 1 shows temperature ranges of the degradation stages, corresponding \%weight loss, maximum thermal decomposition temperature ( $\mathrm{DTG}_{\max }$ ), and \% residual carbon for the selected lignin samples. The TG curve represents \%weight loss as a function of temperature, while the DTG curve represents the rate of weight loss at the corresponding temperature range. The peak maxima of the DTG curve is referred to as DTG $_{\max }$, which represents a single temperature where thermal degradation occurs at the highest rate. Therefore, $\mathrm{DTG}_{\max }$ is considered an essential thermogravimetric parameter to determine and compare the thermal stability of any material.

The weight loss of the lignin samples was initiated due to the evaporation of free and bound water, as observed by a small peak up to $\sim 120^{\circ} \mathrm{C}$ on the DTG curve. This weight loss is considered as the moisture removal or drying stage. The shoulder-like peak in the temperature range of $\sim 120-240^{\circ} \mathrm{C}$ on the DTG curve can be attributed to the breakdown of side-chains, releasing volatiles such as $\mathrm{CO}, \mathrm{CO}_{2}, \mathrm{CH}_{4}$, etc. [23]. This segment is considered the first stage of thermal degradation. Note that lignin degradation in this stage proceeded at a relatively slow rate because both lignins had to overcome their respective glass transition phase, which is a slow process. However, both lignin samples underwent a rapid pyrolytic degradation in the temperature range of $\sim 240-530^{\circ} \mathrm{C}$, as shown by the broad peak on the DTG curve. This is considered the main pyrolysis stage because both lignin samples lost most of their initial weight during this stage. Indulin AT lost about $\sim 37 \%$ weight, whereas alkali lignin lost about $\sim 39 \%$ of its initial weight. This pyrolytic degradation process is associated with the fragmentation of the interunit linkages, releasing monomeric phenols and their derivatives into the vapor phase [18, 24]. Even though both lignin samples had a similar course of thermal degradation and corresponding \%weight loss up to this point, the peak maxima or DTG $_{\max }$ of indulin AT was observed at $395{ }^{\circ} \mathrm{C}$, whereas it was $355^{\circ} \mathrm{C}$ for the alkali lignin. The higher $\mathrm{DTG}_{\max }$ value indicates that indulin AT is thermally more stable compared to alkali lignin. Such 
high thermal stability of the indulin lignin suggests the presence of higher content of C-C linkages, which are very stable bonds and require high temperature to fragment [25]. A very weak shoulder peak was observed at $347^{\circ} \mathrm{C}$ on the DTG curve of indulin AT only, which indicates the coexistence of cellulose and hemicellulose from the pulping process [26]. The third significant degradation for the lignin samples occurred after $\sim 550{ }^{\circ} \mathrm{C}$. The lignin pyrolysis process in this stage involves the decomposition of some aromatic rings [18, 24]. Both lignin samples became highly condensed by forming nonvolatilized char (carbonaceous residue) after heating to $700{ }^{\circ} \mathrm{C}$. During this char formation stage, both lignin samples degraded slowly over a wide temperature range compared to the main pyrolysis stage. However, starting from $\sim 550^{\circ} \mathrm{C}$, indulin AT degraded at a relatively higher rate, as shown by the two hump-like peaks, which are absent in the case of alkali lignin. Such degradation behavior of the lignin samples yielded a lower percentage of residual carbon for indulin AT ( $\sim 41 \%)$ than alkali lignin ( $\sim 47 \%)$. The higher degree of residual carbon content in the alkali lignin suggests its high cross-linking capacity between the aromatic rings (due to re-polymerization at elevated temperature) compared to indulin AT.

\subsubsection{Differential scanning calorimetry (DSC)}

DSC is an excellent thermoanalytical technique for determining glass transition temperature $\left(\mathrm{T}_{\mathrm{g}}\right)$ of amorphous polymers like lignin. Fig. 3(A) represents an overlay of DSC heating curves of the selected lignins showing enthalpy changes in the form of $T_{\mathrm{g}}$ and melting point, and Fig. 3(B) shows an enlarged view of the $T_{\mathrm{g}}$ determination.

During the glass transition, a small change in the baseline is observed on the DSC heating curve rather than a sharp peak because the glass transition of lignin is not a formal phase transition. Therefore, the small changes at 148 and $137^{\circ} \mathrm{C}$ on the DSC baseline were determined to be the $T_{g}$ for indulin AT and alkali lignin, respectively. The lower $T_{g}$ value of alkali lignin suggests that its main chain is more flexible for rotation at a lower temperature. Such a structural feature of alkali lignin also implies its additional capability to form physical cross-linking between the monomers. The $T_{\mathrm{g}}$ values obtained for the selected lignin samples are in agreement with the literature data of various technical lignins [26, 27]. The sharp endothermic peaks at 230 and $193^{\circ} \mathrm{C}$ on the DSC curve are the melting points of indulin AT and alkali lignin. The lower melting point of the alkali lignin suggests that the side chains start to fragment at a lower temperature to release volatiles. Indulin AT having a higher value of $\mathrm{T}_{\mathrm{g}}$ and melting point can be 
deemed to have higher thermal stability; however, they will be less prone to form cross-linking due to the stiffer nature of the main chain compared to alkali lignin.

\subsection{Structural characterization}

\subsubsection{Cupric Oxide $(\mathrm{CuO})$ oxidation}

$\mathrm{CuO}$ oxidation selectively breaks (oxidative cleavage) the ether linkages between the monomeric lignin units instead of breaking the aromatic rings, thus producing various ligninderived phenolic products. The formation of specific phenolic compounds and their relative abundance mainly depend on the specific type of precursor(s) (H/G/S-unit) present in the respective lignin macromolecule. Thus $\mathrm{CuO}$ oxidation can be used to determine the structural moieties (H/G/S) of which the selected lignins are mainly composed of. In this study, the degradation products were extracted with dichloromethane (DCM) and then identified by GCMS. Gas chromatograms of the phenolic products and their chemical structures are shown in Fig. 4 and 5, respectively. The identified phenolic products, molecular weight $\left(\mathrm{M}_{\mathrm{w}}\right)$, moiety types, and their \%relative amounts in terms of peak area are presented in Table 2. Each lignin sample produced five different phenolic products in which vanillin, acetovanillone, guaiacol, vinyl guaiacol were the common products. In addition to these, indulin AT produced homovanillic acid, and alkali lignin produced $p$-cresol. Among all the phenolic compounds, vanillin was the primary degradation product produced from both lignins. The relative amount of vanillin obtained from the lignin samples was very similar and found to be $85.0 \pm 2.2 \%$ and $83.6 \pm 2.8 \%$ for indulin AT and alkali lignin, respectively. Note that all the degradation products except for $p$-cresol have only one methoxy group attached to the monolignol (at C-3 position), which means the resultant degradation products are derived from the coniferyl alcohol (G-moiety), indicating both the lignin samples are mainly composed of G-units. However, $p$-cresol obtained from the alkali lignin does not contain any methoxy group, and hence, belongs to the p-hydroxyphenyl $(\mathrm{H})$ moiety.

\subsubsection{Attenuated total reflectance-Fourier transform infrared (ATR-FTIR) spectroscopy}

ATR-FTIR is an efficient technique for identifying and fingerprinting the characteristic absorption bands of various functional groups and specific phenolic moieties present in the lignin structure. Therefore, the FTIR was mainly utilized to identify the characteristic absorption bands specific to the H, G, or S-moiety present in the selected lignin macromolecules. FTIR was also 
able to provide some valuable information about the structural changes that took place during the respective lignin sample's pulping processes. However, lignin being a complex and amorphous polymer, theoretical data assignment is challenging due to the overlap of multiple vibration modes from different functional groups, especially below the spectral region of $1400 \mathrm{~cm}^{-1}$ [28]. Therefore, the band assignments and data interpretation of indulin AT and alkali lignin were accomplished based on the spectral data of various lignin model compounds and specially produced lignins published in various literature [29, 30]. Fig. 6 shows the FTIR spectra of indulin AT and alkali lignin, and Table 3 shows the major band assignments of the FTIR absorption peaks.

Both lignin samples showed a strong and broad absorption band in the range of 3600-3200 $\mathrm{cm}^{-1}$, which can be attributed to the O-H stretching of the aromatic and aliphatic hydroxyl groups. The bands centered at $\sim 2934 \mathrm{~cm}^{-1}$ can be related to the C-H stretching in methyl $\left(-\mathrm{CH}_{3}\right)$ and methylene ( $=\mathrm{CH}_{2}$ ) groups of the side chains, while the bands at $\sim 2850 \mathrm{~cm}^{-1}$ correspond to the C$\mathrm{H}$ stretching of the aromatic methoxy $\left(-\mathrm{O}-\mathrm{CH}_{3}\right)$ group. However, $\mathrm{C}-\mathrm{H}$ bending vibration in methyl, methylene, and methoxy groups of the phenylpropane unit was observed at $\sim 1451 \mathrm{~cm}^{-1}$. The weak band at $\sim 1783 \mathrm{~cm}^{-1}$, only shown by indulin AT, comes from nonconjugated carbonyl $(\mathrm{C}=\mathrm{O})$ stretching. This suggests indulin AT contains (more) carboxyl groups after the delignification process, which are absent in the case of alkali lignin. Both lignin samples showed characteristic aromatic skeleton (phenylpropane unit) stretching vibrations at $\sim 1591, \sim 1511$, and $\sim 1422 \mathrm{~cm}^{-1}$, and C-H bending vibration in the side chains of the phenylpropane unit at $\sim 1451$ $\mathrm{cm}^{-1}$. These are typical bands for all types of lignins and suggest that aromatic structures of the selected lignins remained reasonably unaffected by the respective pulping process. The weak absorption peak focused at $\sim 1366 \mathrm{~cm}^{-1}$ appeared only in the case of indulin AT, which can be linked to the non-etherified phenolic OH groups produced from the cleavage of $\beta-\mathrm{O}-4$ and $\alpha-\mathrm{O}-4$ linkages during the delignification process $\left[{ }^{31}\right]$. Such observation is suggestive of extensive phenol-ether cleavage of indulin AT during the pulping process. Both lignin samples showed some typical characteristic absorption bands specific to the lignin moiety. For example, the absorption band focused at $\sim 1264 \mathrm{~cm}^{-1}$ can be attributed to the guaiacyl (G) unit plus $\mathrm{C}=\mathrm{O}$ stretch (guaiacyl ring breathing), the band at $\sim 1126 \mathrm{~cm}^{-1}$ was due to the $\mathrm{C}-\mathrm{H}$ in-plane deformation of the guaiacyl (G) unit, while the bands focused at $\sim 854$ and $\sim 813 \mathrm{~cm}^{-1}$ were specific to the $\mathrm{C}-\mathrm{H}$ out-of-plane vibrations in position 2, 5 and 6 of the guaiacyl $(\mathrm{G})$ unit. The 
characteristic syringyl (S) bands (1326, 1115, and $825 \mathrm{~cm}^{-1}$ ) were not observed for either of the lignin samples. Observation of multiple absorption bands specific to the G-moiety indicates that the selected lignins were mainly produced from softwood feedstock rather than hardwood or dicotyl crops [30].

\subsubsection{Proton Nuclear Magnetic Resonance $\left({ }^{1} H\right.$ NMR) spectroscopic analysis}

The macromolecular structure of the selected lignin samples was further studied using ${ }^{1} \mathrm{H}$ NMR spectroscopy. However, ${ }^{\text {the }}{ }^{1} \mathrm{H}$ NMR spectrum of lignin suffers from signal overlapping due to its complex and heterogeneous macromolecular matrix. Therefore, rather than attempting to unravel the detailed molecular structure, the ${ }^{1} \mathrm{H}$ NMR study was mainly intended to obtain information about the structural changes incurred to the lignin samples due to the respective delignification process. To this end, several key chemical functionalities, including phenolic hydroxyl (-OH) group, $\beta-\mathrm{O}-4$ linkage, methoxyl (-OCH $)$ group, and monolignol moiety $(\mathrm{H} / \mathrm{G} / \mathrm{S})$ of the lignin macromolecule were quantitively examined. Fig. 7(A) and (B) show ${ }^{1} \mathrm{H}$ NMR spectra of indulin AT and alkali lignin, respectively. The chemical shift values and corresponding signal assignments are summarized in Table 4, and quantification of some vital functional groups are shown in Table 5. The chemical shift values assigned to the functional groups and linkages are based on the various lignin model compounds published in various literature and database [32-34]. The ${ }^{1} \mathrm{H}$ NMR signals from 6.0 to $7.8 \mathrm{ppm}$ can be attributed to the aromatic protons of the guaiacol (G-ring) moiety. The specific signals related to the three aromatic protons were detected at 6.76, 6.95, and $7.14 \mathrm{ppm}$. Table 5 shows that the quantity of all the aromatic protons in the Gring is $3.28 \mathrm{mmol} / \mathrm{g}$ for alkali lignin, whereas it is $6.55 \mathrm{mmol} / \mathrm{g}$ for indulin AT. This proves that indulin AT has a higher content of guaiacyl units (G-rings) in its structure than the alkali lignin. The broad spectral region in the range of 8.4-9.4 ppm is specific to the proton in the phenolic hydroxyl group. Indulin AT (3.80 mmol/g) has a higher content of free phenolic hydroxyls compared to alkali (1.16 mmol.g $\left.{ }^{-1}\right)$, which suggests an ample cleavage of alkyl-aryl ether linkages ( $\beta-\mathrm{O}-4, \alpha-\mathrm{O}-4$, etc.) during the cooking process, leaving a lot of non-etherified phenolic $\mathrm{OH}$ groups [19]. The ${ }^{1} \mathrm{H}$ NMR spectrum of alkali lignin also shows methoxyl groups $\left(-\mathrm{OCH}_{3}\right)$ and $\beta$ O-4 linkages centered at 3.40 and $3.90 \mathrm{ppm}$, respectively. The quantity of methoxyl groups in the alkali lignin was measured to be $1.42 \mathrm{mmol} / \mathrm{g}$, whereas the quantity of $\beta$-O-4 linkages was 0.95 $\mathrm{mmol} / \mathrm{g}$. Unfortunately, such chemical functionalities in indulin AT could not be identified due to the severe peak overlapping from a very intense and broad peak ranging from 2.8 to $4.5 \mathrm{ppm}$. 
Observation of such a broad peak in this region can be attributed to cellulose and hemicellulose in the indulin lignin sample.

\section{Conclusions}

A combination of thermal (TGA; DSC), degradative (CuO oxidation), and non-degradative (ATR-FTIR; ${ }^{1} \mathrm{H}$ NMR) techniques were utilized to reveal the overall macromolecular characteristics of two technical lignins, namely indulin AT and alkali lignin. The results obtained from these characterization studies agree with each other and suggest that both lignin samples are almost exclusively composed of guaiacyl (G-type) moiety in terms of monolignol composition. However, there are some subtle structural differences in the molecular matrices of the lignin samples due to the difference in their respective delignification process. Such structural differences alter the overall properties of the selected lignins, making one lignin more suitable than the other for various high-value applications. TGA and DSC analyses revealed that indulin AT having higher $\mathrm{DTG}_{\max }$ and $\mathrm{T}_{\mathrm{g}}$ value is thermally more stable, most likely due to higher amounts of C-C linkages. CuO oxidation (see Table 2) and ${ }^{1} \mathrm{H}$ NMR (see Table 5) studies revealed that indulin AT has additional guaiacyl units (G-ring) in its structure compared to alkali lignin. The C-C bonds between the guaiacyl units at the C-5 position are very stable [28]; hence, indulin AT would be more recalcitrant towards further depolymerization into valuable phenolic products than the alkali lignin. FTIR and ${ }^{1} \mathrm{H}$ NMR study also revealed that indulin AT has a higher content of phenolic hydroxyls, which implies that indulin AT has undergone an extensive cleavage of alpha-aryl and $\beta$-aryl ether bonds due to a harsher delignification process. Such high phenolic hydroxyls in indulin AT would allow the activation of free ring position, making indulin AT more reactive [35] and can be exploited for lignin functionalization in various high-value applications such as synthesis of phenol-formaldehyde resin. However, TGA and ${ }^{1} \mathrm{H}$ NMR studies also hinted at the presence of cellulose and hemicellulose in the indulin, making this lignin sample less pure than the alkali lignin. Overall, the alkali lignin's chemical composition was closer to its native structure than indulin AT, which suggests alkali lignin underwent fewer structural modifications during the delignification process. Thus, detailed structural information obtained from this study would allow rapid and efficient utilization of these two technical lignins in various high-value applications. 


\section{- AUTHOR INFORMATION}

Corresponding Author*

Tel.: +16056886361,

E-mail: douglas.raynie@sdstate.edu

\section{Notes}

The authors declare no competing financial interest.

\section{- ACKNOWLEDGEMENTS}

This study was supported by the National Science Foundation EPSCoR Track II Dakota BioCon project for the North and South Dakota (Grant No. IIA-1355466 and IIA-1330842). The authors are grateful to the Department of Pharmaceutical Sciences (SDSU) for their permission to use the FT-IR facility.

\section{References}

1. Ahvazi, B.; Cloutier, E. r.; Wojciechowicz, O.; Ngo, T.-D., Lignin profiling: a guide for selecting appropriate lignins as precursors in biomaterials development. ACS Sustainable Chemistry \& Engineering 2016, 4 (10), 5090-5105.

2. Nashawi, I. S.; Malallah, A.; Al-Bisharah, M., Forecasting world crude oil production using multicyclic Hubbert model. Energy \& Fuels 2010, 24 (3), 1788-1800.

3. Den, W.; Sharma, V. K.; Lee, M.; Nadadur, G.; Varma, R. S., Lignocellulosic biomass transformations via greener oxidative pretreatment processes: access to energy and value-added chemicals. Frontiers in chemistry 2018, 6, 141.

4. Fengel, D.; Wegener, G., Wood: chemistry, ultrastructure, reactions. Walter de Gruyter: 1983.

5. Hill, C. A., Wood modification: chemical, thermal and other processes. John Wiley \& Sons: 2007; Vol. 5. 
6. Paone, E.; Tabanelli, T.; Mauriello, F., The rise of lignin biorefinery. Current Opinion in Green and Sustainable Chemistry 2020, 24, 1-6.

7. $\quad$ Laurichesse, S.; Avérous, L., Chemical modification of lignins: Towards biobased polymers. Progress in polymer science 2014, 39 (7), 1266-1290.

8. Sen, S.; Patil, S.; Argyropoulos, D. S., Thermal properties of lignin in copolymers, blends, and composites: a review. Green Chemistry 2015, 17 (11), 4862-4887.

9. Bajwa, D.; Pourhashem, G.; Ullah, A.; Bajwa, S., A concise review of current lignin production, applications, products and their environmental impact. Industrial Crops and Products 2019, 139, 111526.

10. Lora, J. H.; Glasser, W. G., Recent industrial applications of lignin: a sustainable alternative to nonrenewable materials. Journal of Polymers and the Environment 2002, 10 (1-2), 39-48.

11. Lai, C.; Zhou, Z.; Zhang, L.; Wang, X.; Zhou, Q.; Zhao, Y.; Wang, Y.; Wu, X.-F.; Zhu, Z.; Fong, H., Free-standing and mechanically flexible mats consisting of electrospun carbon nanofibers made from a natural product of alkali lignin as binder-free electrodes for highperformance supercapacitors. Journal of Power Sources 2014, 247, 134-141.

12. Cinelli, P.; Anguillesi, I.; Lazzeri, A., Green synthesis of flexible polyurethane foams from liquefied lignin. European Polymer Journal 2013, 49 (6), 1174-1184.

13. Nevárez, L. A. M.; Casarrubias, L. B.; Celzard, A.; Fierro, V.; Muñoz, V. T.; Davila, A. C.; Lubian, J. R. T.; Sánchez, G. G., Biopolymer-based nanocomposites: effect of lignin acetylation in cellulose triacetate films. Science and technology of advanced materials 2011, 12 (4), 045006.

14. Deepa, A. K.; Dhepe, P. L., Lignin depolymerization into aromatic monomers over solid acid catalysts. ACS Catalysis 2015, 5 (1), 365-379.

15. Reale S Fau - Di Tullio, A.; Di Tullio A Fau - Spreti, N.; Spreti N Fau - De Angelis, F.; De Angelis, F., Mass spectrometry in the biosynthetic and structural investigation of lignins. (0277-7037 (Print)).

16. Boeriu, C. G.; Fiţigău, F. I.; Gosselink, R. J.; Frissen, A. E.; Stoutjesdijk, J.; Peter, F., Fractionation of five technical lignins by selective extraction in green solvents and characterisation of isolated fractions. Industrial Crops and Products 2014, 62, 481-490. 
17. Hatakeyama, T.; Hatakeyama, H., Thermal properties of green polymers and biocomposites. Springer Science \& Business Media: 2006; Vol. 4.

18. Sun, R.; Tomkinson, J.; Jones, G. L., Fractional characterization of ash-AQ lignin by successive extraction with organic solvents from oil palm EFB fibre. Polymer Degradation and Stability 2000, 68 (1), 111-119.

19. Gellerstedt, G.; Lindfors, E.-L., Structural changes in lignin during kraft pulping. Holzforschung-International Journal of the Biology, Chemistry, Physics and Technology of Wood 1984, 38 (3), 151-158.

20. Olivares, M.; Guzman, J.; Natho, A.; Saavedra, A., Kraft lignin utilization in adhesives. Wood Science and Technology 1988, 22 (2), 157-165.

21. Yoshida, H.; Mörck, R.; Kringstad, K. P.; Hatakeyama, H., Fractionation of kraft lignin by successive extraction with organic solvents. II. Thermal properties of kraft lignin fractions. Holzforschung-International Journal of the Biology, Chemistry, Physics and Technology of Wood 1987, 41 (3), 171-176.

22. Pearl, I. A., Studies on Lignin and Related Products. XIII. 1 Oxidations of Lignin Model Compounds and their Significance to Lignin Structure2. Journal of the American Chemical Society 1956, 78 (21), 5672-5674.

23. Chen, G.; Leung, D., Experimental investigation of biomass waste,(rice straw, cotton stalk, and pine sawdust), pyrolysis characteristics. Energy Sources 2003, 25 (4), 331-337. 24. El-Saied, H.; Nada, A.-A. M., The thermal behaviour of lignins from wasted black pulping liquors. Polymer degradation and stability 1993, 40 (3), 417-421.

25. Jakab, E.; Faix, O.; Till, F., Thermal decomposition of milled wood lignins studied by thermogravimetry/mass spectrometry. Journal of Analytical and Applied Pyrolysis 1997, 40, 171186.

26. Saito, T.; Perkins, J. H.; Vautard, F.; Meyer, H. M.; Messman, J. M.; Tolnai, B.; Naskar, A. K., Methanol fractionation of softwood kraft lignin: Impact on the lignin properties. ChemSusChem 2014, 7 (1), 221-228.

27. Dodd, A. P.; Kadla, J. F.; Straus, S. K., Characterization of fractions obtained from two industrial softwood kraft lignins. ACS Sustainable Chemistry \& Engineering 2015, 3 (1), 103110. 
28. El Mansouri, N. E.; Yuan, Q.; Huang, F., Characterization of alkaline lignins for use in phenol-formaldehyde and epoxy resins. BioResources 2011, 6 (3), 2647-2662.

29. Faix, O., Fourier transform infrared spectroscopy. In Methods in lignin chemistry, Springer: 1992; pp 83-109.

30. Boeriu, C. G.; Bravo, D.; Gosselink, R. J.; van Dam, J. E., Characterisation of structuredependent functional properties of lignin with infrared spectroscopy. Industrial crops and products 2004, 20 (2), 205-218.

31. Tejado, A.; Pena, C.; Labidi, J.; Echeverria, J.; Mondragon, I., Physico-chemical characterization of lignins from different sources for use in phenol-formaldehyde resin synthesis. Bioresource technology 2007, 98 (8), 1655-1663.

32. Li, S.; Lundquist, K., A new method for the analysis of phenolic groups in lignins by proton NMR spectrometry. Nordic Pulp \& Paper Research Journal 1994, 9 (3), 191-5.

33. Lundquist, K., NMR studies of lignins. 5. Investigation of nonderivatized spruce and birch lignin by proton NMR spectroscopy. Acta Chemica Scandinavica, Series B: Organic Chemistry and Biochemistry 1981, B35 (7), 497-501.

34. Lundquist, K.; Olsson, T., NMR studies of lignins. 1. Signals due to protons in formyl groups. Acta Chemica Scandinavica, Series B: Organic Chemistry and Biochemistry 1977, 31 (9), 788-92.

35. Stolker, A.; Van Schoonhoven, J.; De Vries, A.; Bobeldijk-Pastorova, I.; Vaes, W.; Van Den Berg, R., Determination of cannabinoids in cannabis products using liquid chromatographyion trap mass spectrometry. Journal of Chromatography A 2004, 1058 (1-2), 143-151. 


\section{List of Tables and Figures:}

\section{Tables:}

Table 1. Various degradation stages and corresponding \%weight loss, DTG $_{\max }$, and \% residual carbon of indulin AT and alkali lignin

Table 2. Identified products of $\mathrm{CuO}$ degradation, molecular weight $\left(\mathrm{M}_{\mathrm{w}}\right)$, moiety type, and their percent relative abundance

Table 3. Assignment of ATR-FTIR absorption bands of indulin AT and alkali lignin

Table 4. ${ }^{1} \mathrm{H}$ NMR signal assignments for indulin AT and alkali lignin

Table 5. Quantification of some key chemical functionalities in indulin AT and alkali lignin using ${ }^{1} \mathrm{H}$ NMR

\section{Figures:}

Fig 1. Monomeric building blocks of lignin.

Fig. 2. (A) TG and (B) DTG thermograms of indulin AT and alkali lignin obtained from TGA analysis.

Fig. 3. (A) DSC heating curve of indulin AT and alkali lignin. (B) Enlarged view of DSC curve for $\mathrm{T}_{\mathrm{g}}$ determination.

Fig. 4. Gas chromatograms of indulin $\mathrm{AT}$ and alkali lignin obtained from $\mathrm{CuO}$ oxidation analysis.

Fig 5. Chemical structures of the phenolic products obtained from the $\mathrm{CuO}$ degradation study of the selected lignins.

Fig. 6. ATR-FTIR spectra of indulin AT and alkali lignin.

Fig. 7. ${ }^{1} \mathrm{H}$ NMR spectra of (A) indulin AT and (B) alkali lignin. 
Table 1. Various degradation stages and corresponding \%weight loss, DTG $_{\max }$, and \% residual carbon of indulin AT and alkali lignin

\begin{tabular}{|c|c|c|c|c|c|}
\hline $\begin{array}{l}\text { Lignin } \\
\text { sample }\end{array}$ & $\begin{array}{l}\text { Degradation } \\
\text { stage }\end{array}$ & $\begin{array}{l}\text { Temperature } \\
\text { range }\left({ }^{\circ} \mathrm{C}\right)\end{array}$ & $\begin{array}{l}\text { \%Weight } \\
\text { loss }\end{array}$ & $\begin{array}{l}\text { DTG }_{\max } \\
\left({ }^{\circ} \mathrm{C}\right)\end{array}$ & $\begin{array}{l}\text { Residual C } \\
\text { (\%) }\end{array}$ \\
\hline \multirow{5}{*}{ Indulin AT } & Drying & Up to 120 & $1.89 \pm 0.33$ & \multirow{5}{*}{395} & \multirow{5}{*}{$40.81 \pm 1.12$} \\
\hline & $1 \mathrm{st}$ & $\sim 120-240$ & $12.44 \pm 0.56$ & & \\
\hline & $2^{\text {nd }}$ (pyrolysis) & $\sim 240-530$ & $36.76 \pm 0.87$ & & \\
\hline & $3^{\text {rd }}$ & $\sim 550-700$ & $8.23 \pm 0.44$ & & \\
\hline & Charring & After 700 & $-*$ & & \\
\hline \multirow{5}{*}{ Alkali } & Drying & Up to 120 & $1.56 \pm 0.45$ & \multirow{5}{*}{355} & \multirow{5}{*}{$47.22 \pm 0.98$} \\
\hline & $1 \mathrm{st}$ & $\sim 120-240$ & $10.17 \pm 0.88$ & & \\
\hline & $2^{\text {nd }}$ (pyrolysis) & $\sim 240-530$ & $38.82 \pm 0.73$ & & \\
\hline & $3^{\text {rd }}$ & $\sim 550-700$ & $2.23 \pm 0.18$ & & \\
\hline & Charring & After 700 & -* & & \\
\hline
\end{tabular}

*- Not observed/negligible 
Table 2. Identified products of $\mathrm{CuO}$ degradation, molecular weight $\left(\mathrm{M}_{\mathrm{w}}\right)$, moiety type, and their percent relative abundance

\begin{tabular}{cclccc}
\hline & $\begin{array}{c}\text { Peak } \\
\text { no. }\end{array}$ & Identified products & $\mathbf{M}_{\mathbf{w}}$ & $\begin{array}{c}\text { Moiety } \\
\text { type }\end{array}$ & $\begin{array}{c}\text { Relative } \\
\text { amount (\%) }\end{array}$ \\
\hline \multirow{3}{*}{ Indulin } & 1 & Guaiacol & 124.1 & $\mathrm{G}$ & $4.4 \pm 0.8$ \\
AT & 2 & $p$-vinyl guaiacol & 150.2 & $\mathrm{G}$ & $1.1 \pm 0.3$ \\
& 3 & Vanillin & 152.1 & $\mathrm{G}$ & $85.0 \pm 2.2$ \\
& 4 & Acetovanillone & 166.2 & $\mathrm{G}$ & $6.3 \pm 1.1$ \\
& 5 & Homovanillic acid & 182.2 & $\mathrm{G}$ & $3.2 \pm 0.5$ \\
\hline \multirow{3}{*}{ Alkali } & 1 & Guaiacol & 124.1 & $\mathrm{G}$ & $3.0 \pm 0.7$ \\
& 2 & p-Cresol & 108.1 & $\mathrm{H}$ & $2.4 \pm 0.2$ \\
& 3 & p-vinyl guaiacol & 150.2 & $\mathrm{G}$ & $2.0 \pm 0.5$ \\
& 4 & Vanillin & 152.1 & $\mathrm{G}$ & $83.6 \pm 2.8$ \\
& 5 & Acetovanillone & 166.2 & $\mathrm{G}$ & $9.0 \pm 1.6$ \\
\hline
\end{tabular}


Table 3. Assignment of ATR-FTIR absorption bands of indulin AT and alkali lignin

\begin{tabular}{clc}
\hline Peak $\left.\mathbf{( c m}^{-\mathbf{1}}\right)$ & Band assignment & Moiety \\
\hline 3342 & Phenolic and aliphatic O-H stretching & $-*$ \\
2934 & C-H stretching $\left(\mathrm{CH}_{3}\right.$ and $\mathrm{CH}_{2}$ groups) & $-*$ \\
2850 & C-H stretching $\left(\mathrm{OCH}_{3}\right.$ groups) & $-*$ \\
1783 & C=O stretch (in unconjugated ketone, carbonyl, and ester & $-*$ \\
& groups) & $-*$ \\
1591 & C-C stretching (aromatic skeleton) & $-*$ \\
1511 & C-C stretching (aromatic skeleton) & $-*$ \\
1451 & C-H deformation (asymmetric in $-\mathrm{CH}_{3}$ and $\left.-\mathrm{CH}_{2}\right)$ & $-*$ \\
1422 & C-C stretching (aromatic skeleton) with C-H in-plane & \\
& deformation & $-*$ \\
1366 & O-H in-plane deformation (phenolic OH) & G \\
1264 & Guaiacyl (G) unit plus C=O stretching & $-*$ \\
1213 & C-O(H) + C-O(Ar) stretching & G \\
1126 & In-plane deformation of Ar C-H (G) & $-*$ \\
1078 & C-O stretching and C-O deformation & $-*$ \\
1030 & C-O(H) + C-O(C) stretching (first-order aliphatic OH and & \\
854 & ether) & G \\
813 & C-H out-of-plane vibration in position 2, 5, and 6 of G unit & G \\
\hline
\end{tabular}

*- Not observed 
Table 4. ${ }^{1} \mathrm{H}$ NMR signal assignments for indulin AT and alkali lignin

\begin{tabular}{ll}
\hline Peak (ppm) & Functional Groups \\
\hline 1.20 & Hydrocarbon contaminant \\
2.50 & DMSO-d 6 solvent \\
3.40 & Proton in Methoxyl group \\
3.7 & Cellulose/hemicellulose \\
3.80 & Proton in hydroxyl group (aliphatic) \\
3.90 & ß-O-4 linkage \\
5.12 & 1,3,5-trioxane (Internal standard) \\
$6.0-7.8$ & Aromatic H in G unit \\
$8.4-9.4$ & Proton in phenolic groups \\
9.78 & Formyl protons \\
\hline
\end{tabular}


Table 5. Quantification of some key chemical functionalities in indulin AT and alkali lignin using ${ }^{1} \mathrm{H}$ NMR

\begin{tabular}{lll}
\hline Functional group & $\begin{array}{l}\text { Indulin AT } \\
\text { (mmol/g) }\end{array}$ & $\begin{array}{l}\text { Alkali } \\
\text { (mmol/g) }\end{array}$ \\
\hline $\mathrm{H}$ in $-\mathrm{OCH}_{3}$ & $-*$ & 1.42 \\
$\beta$-O-4 linkage & $-*$ & 0.95 \\
Aromatic protons in G-ring & 6.55 & 3.28 \\
$\mathrm{H}$ in Phenolic OH & 3.80 & 1.16 \\
\hline *- Not observed & &
\end{tabular}

*- Not observed 


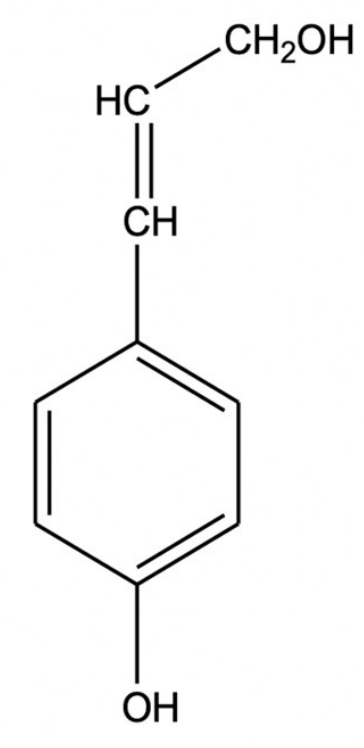

p-hydroxycinnamyl alcohol (H)

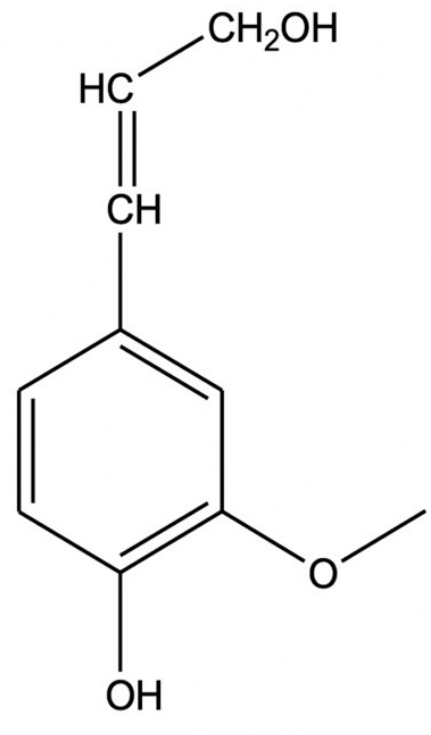

coniferyl alcohol (G)

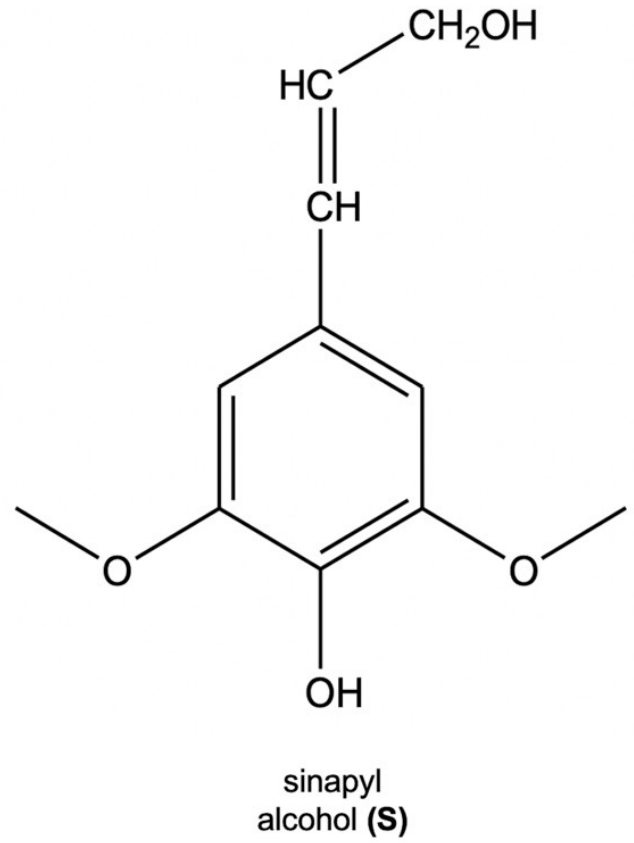

Fig 2. Monomeric building blocks of lignin. 

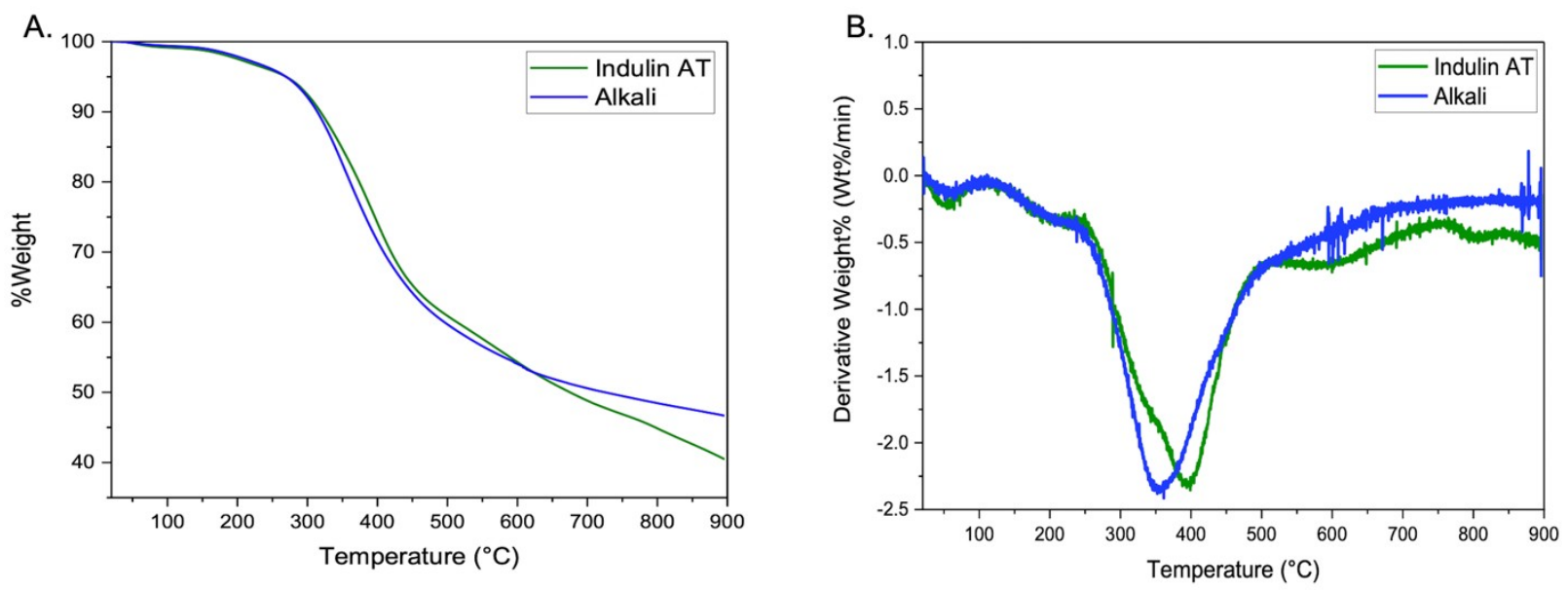

Fig. 2. (A) TG and (B) DTG thermograms of indulin AT and alkali lignin obtained from TGA analysis. 

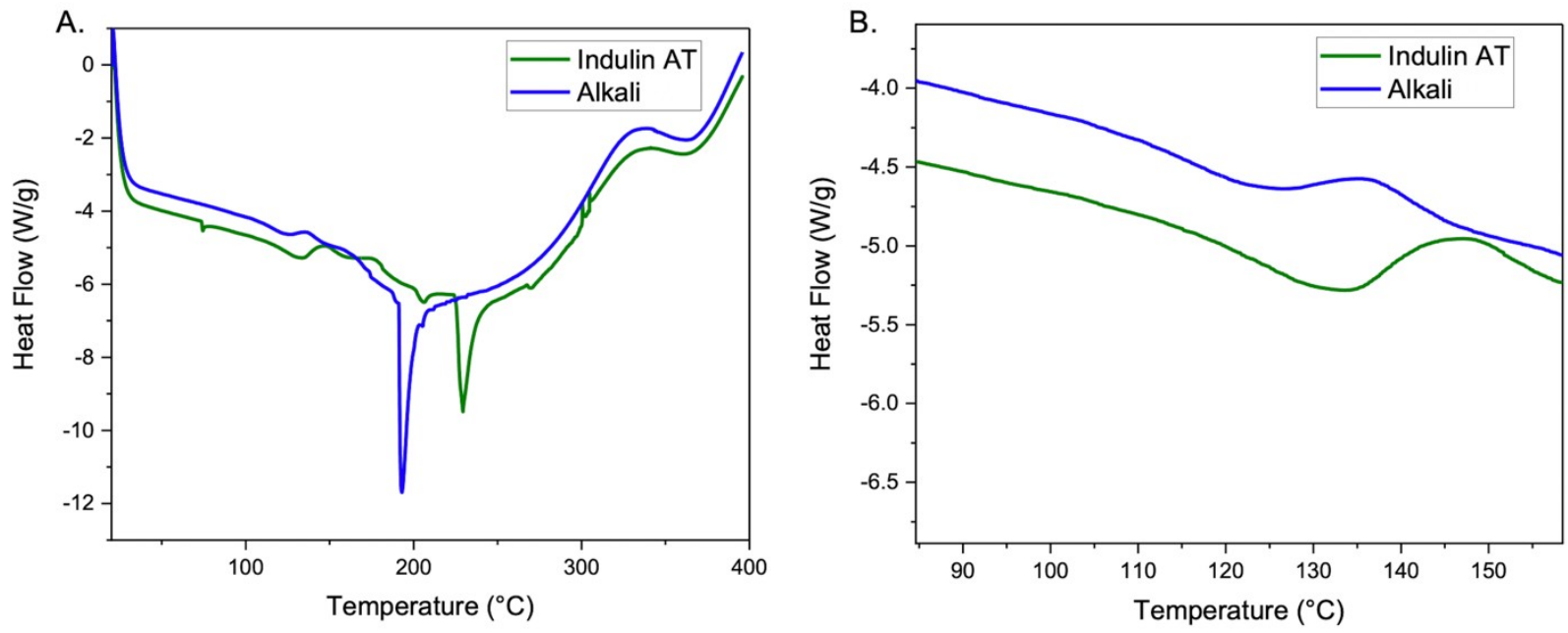

Fig. 3. (A) DSC heating curve of indulin AT and alkali lignin. (B) Enlarged view of DSC curve for $T_{g}$ determination. 

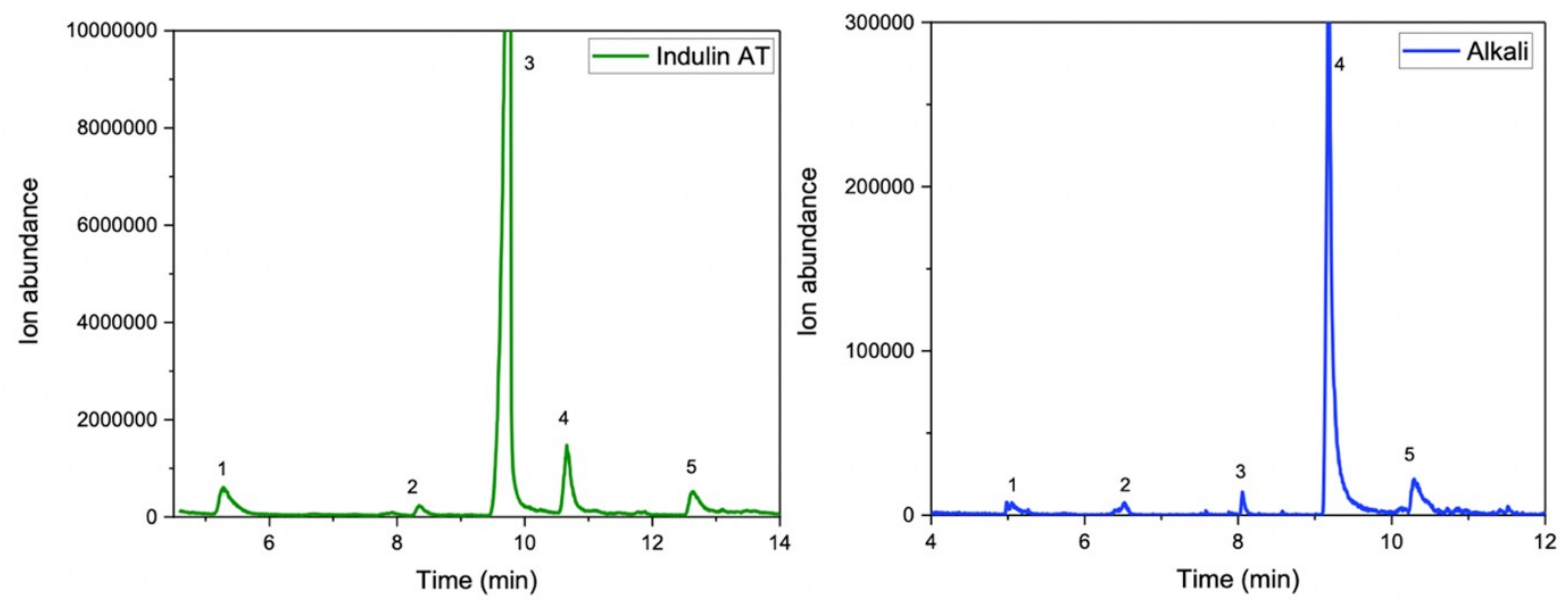

Fig. 4. Gas chromatograms of indulin AT and alkali lignin obtained from $\mathrm{CuO}$ oxidation analysis. 
<smiles>COc1ccccc1O</smiles>

Guaiacol<smiles>Cc1ccc(O)cc1</smiles>

p-cresol<smiles>COc1cc(C=O)ccc1O</smiles>

Vanillin<smiles>COc1cc(C(C)=O)ccc1O</smiles>

Acetovanillone<smiles>C=Cc1ccc(O)c(OC)c1</smiles>

p-vinyl guaiacol<smiles>COc1cc(CC(=O)O)ccc1O</smiles>

Homovanillic acid

Fig 5. Chemical structures of the phenolic products obtained from the $\mathrm{CuO}$ degradation study of the selected lignins. 


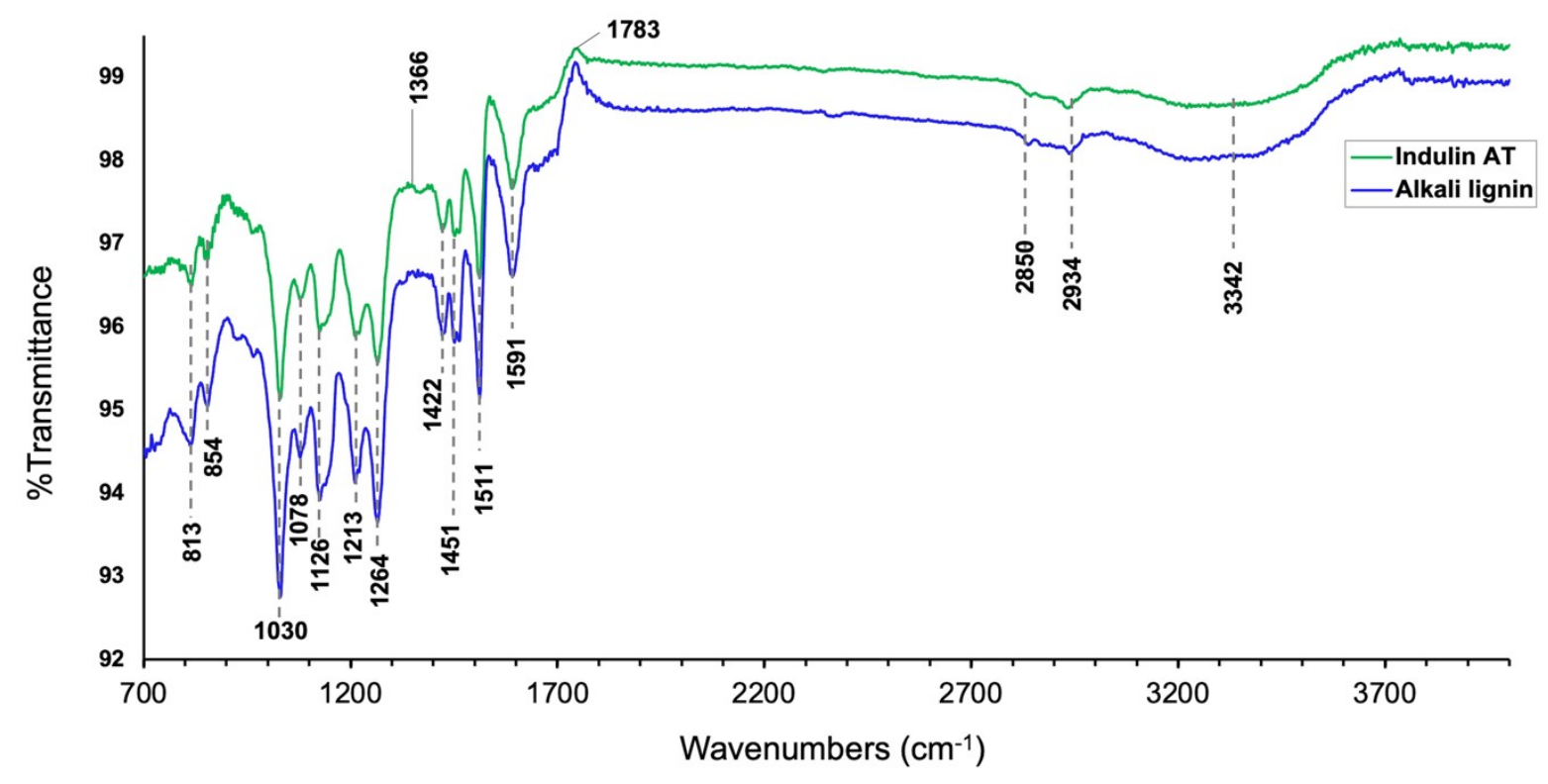

Fig. 6. FTIR spectra of indulin AT and alkali lignin. 


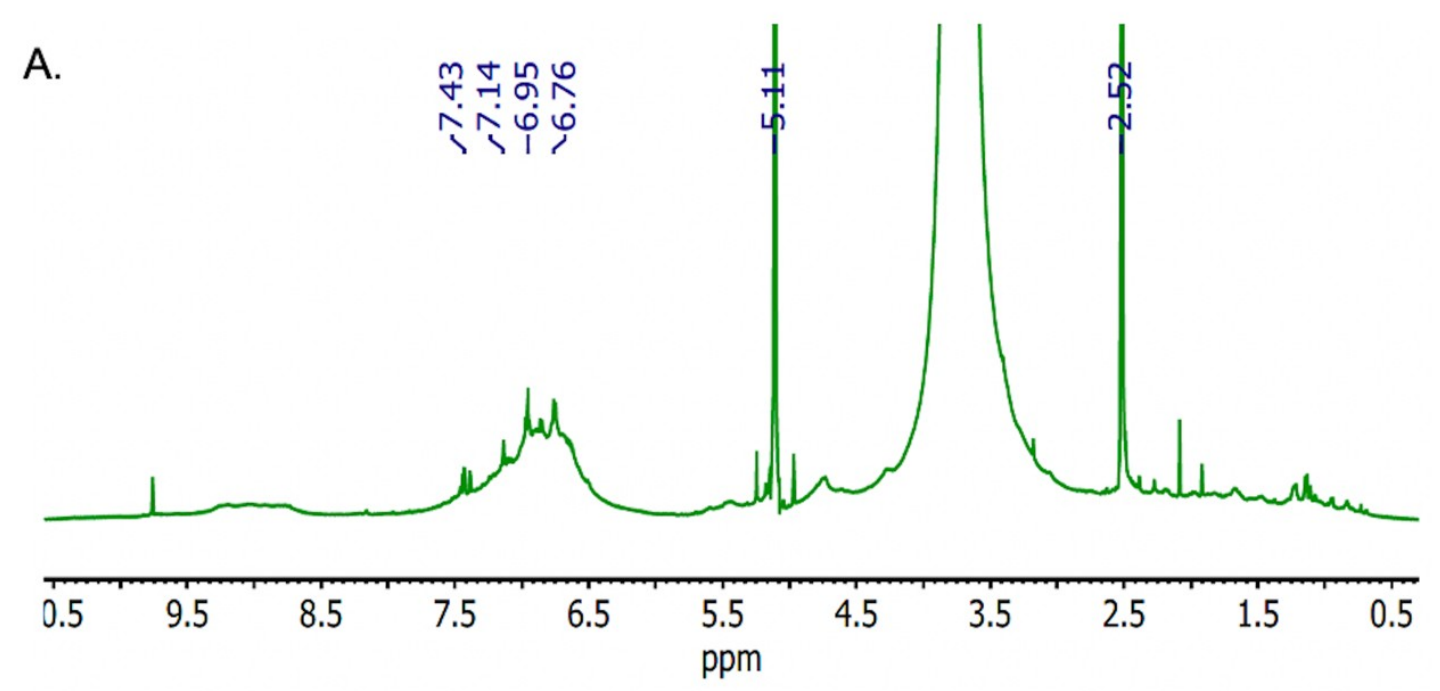

B.

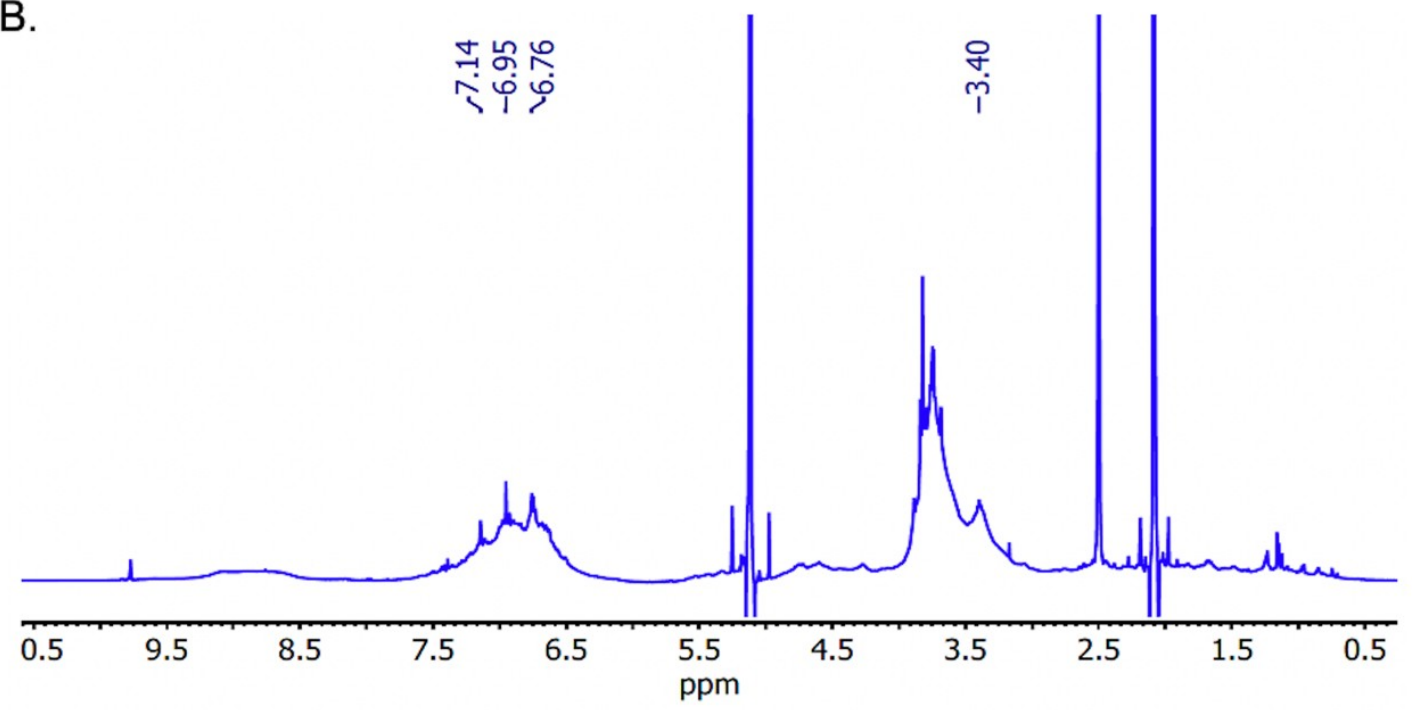

Fig. 7. ${ }^{1} \mathrm{H}$ NMR spectra of (A) indulin AT and (B) alkali lignin. 
